\title{
Measurement and Modeling of the Transfer Function of a Monolithic SOA-EA 2R- Regenerator
}

\author{
Christiansen, Lotte Jin; Öhman, Filip; Kjær, Rasmus; Mørk, Jesper; Yvind, Kresten
}

Published in:

CLEO/QELS 2005 Technical Digest CD-Rom

Publication date:

2005

Document Version

Publisher's PDF, also known as Version of record

Link back to DTU Orbit

Citation $(A P A)$ :

Christiansen, L. J., Öhman, F., Kjær, R., Mørk, J., \& Yvind, K. (2005). Measurement and Modeling of the Transfer Function of a Monolithic SOA-EA 2R-Regenerator. In CLEO/QELS 2005 Technical Digest CD-Rom IEEE.

\section{General rights}

Copyright and moral rights for the publications made accessible in the public portal are retained by the authors and/or other copyright owners and it is a condition of accessing publications that users recognise and abide by the legal requirements associated with these rights.

- Users may download and print one copy of any publication from the public portal for the purpose of private study or research.

- You may not further distribute the material or use it for any profit-making activity or commercial gain

- You may freely distribute the URL identifying the publication in the public portal 


\title{
Measurement and Modeling of the Transfer Function of a Monolithic SOA-EA 2R-Regenerator
}

\author{
Lotte Jin Christiansen, Filip Öhman, Rasmus Kjar, Jesper Mørk and Kresten Yvind \\ Research Center COM, DTU, Build. 345v, DK-2800 Kgs. Lyngby, Denmark \\ fo@.com.dtu.dk
}

Abstract: We have measured steep power transfer characteristics with tunable threshold for a monolithic 2Rregenerator combining amplifying and absorbing sections. Modeling results explain the basic characteristics. (C) 2005 Optical Society of America

OCIS codes: (130.0250) Optoelectronics, (230.1150) All-optical devices

\section{Introduction}

The concept of $2 \mathrm{R}$ regeneration is based on the existence of components with nonlinear, step-like power transfer functions [1]. Previously we suggested a new type of regenerator which cascades semiconductor optical amplifiers (SOAs) and electro-absorbers (EAs), see Fig. 1, to implement a nonlinear transfer function [2,3]. Compared to interferometer-based schemes, the device is much simpler, both in terms of fabrication and electrical control, and further avoids the requirements of interferometric stability. The regenerative properties were verified in a system experiment [2], but so far the dependence on the material parameters of the different sections and the bias conditions has not been investigated in detail. We investigate here, experimentally and through modeling, how the cascading of sections shapes up the transfer function and we establish how the threshold can be tuned by varying the bias conditions. The tunability of the threshold is, we believe, an important feature for a practical 2R-regenerator.

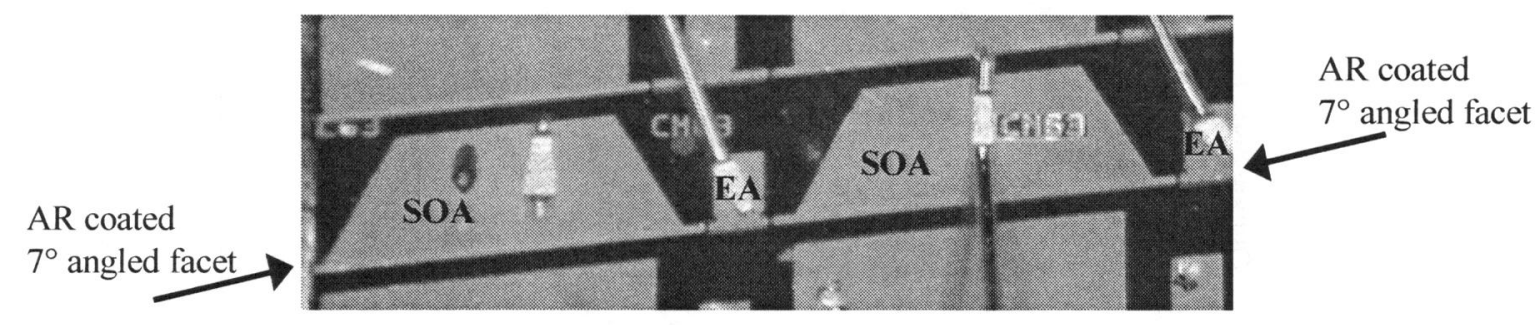

Fig. 1. A photo of the examined device consisting of two concatenated SOA-EA sections.

\section{The measurement setup and model}

The SOA-EA 2R regenerator utilizes the saturable characteristic of the absorbing [4] and amplifying elements in order to achieve reshaping at low as well as high power levels. The device can be fabricated on the basis of a single long waveguide section with the same active region, where the electrode is separated into sections that are forward or reverse biased for the SOA and EA sections, respectively. More design freedom is achieved if re-growth is used to allow individual bandgaps for the different sections. Both device types have been fabricated and showed nonlinear transfer functions. It is important to note that for all designs the SOA must be placed at the input side in order to minimize the noise figure of the cascaded sections.

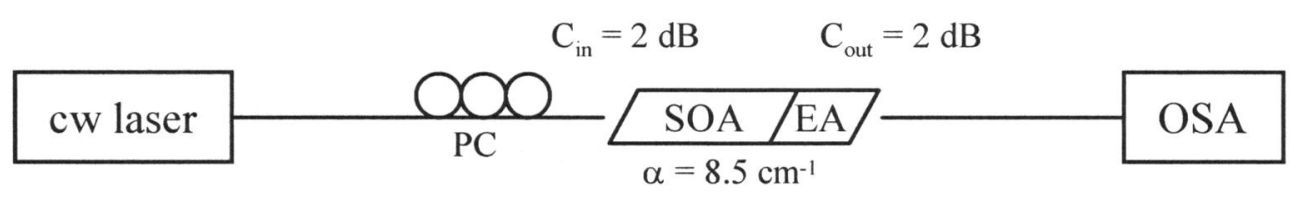

Fig. 2. Schematic illustration of the cw measurement setup with the coupling losses $\mathrm{C}$, and internal losses $\alpha$. Facet losses are negligible due to AR coatings and angled facets. Polarization controller (PC). Optical spectrum analyzer (OSA).

By performing CW measurements on a single SOA-EA section we were able to extract the four very important parameters; gain, absorption, saturation output power of the SOA and saturation input power of the EA. Firstly, the 


\section{CMGG5}

SOA was characterized by omitting the EA by biasing it at transparency. It is important to correctly estimate the optical power levels at the SOA input and output, in order to find the modal gain and SOA output power, which at the same time constitutes the input power to the EA. Hence, all the sources of optical loss have been identified and estimated, and are shown in Fig. 2. After measuring the transfer functions of the sole SOA, the additional loss introduced when applying reverse bias to the EA section is found. The EA absorption and saturation input powers were measured at different applied reverse biases. The obtained values for gain, absorption and saturation were used for the modeling results to be presented.

The SOA-EA device is modeled by solving a set of standard rate equations for the steady state situation. Both the amplifier and absorber use a similar type model but with different parameters, e.g. reflecting the reduction of the lifetime in the EA sections due to carrier-sweep out by the applied field. The gain and absorption are assumed to be linear functions of the carrier density and the carrier lifetimes are assumed power independent.

\section{Results}

In Fig. 3 the measured transfer functions of an SOA-EA regenerator consisting of respectively one and two concatenated SOA-EA sections are shown. The active material consists of five $7.2 \mathrm{~nm}$ thick compressively strained InGaAsP quantum wells in a strain compensated structure, having gain peak at $1535 \mathrm{~nm}$ and a gain bandwidth of 60 $\mathrm{nm}$. The lengths of the SOA sections are $545 \mu \mathrm{m}$ and the lengths of the EA sections are $120 \mu \mathrm{m}$. The bias current to each SOA is $100 \mathrm{~mA}$ and the reverse bias of the first EA is varied between 0 and 0.75 volts, the second EA is unbiased. The improvement in steepness, i.e. regenerative characteristic, when adding an additional pair of SOAEAs is clearly seen. The results also show how to easily change the threshold by varying the EA bias.

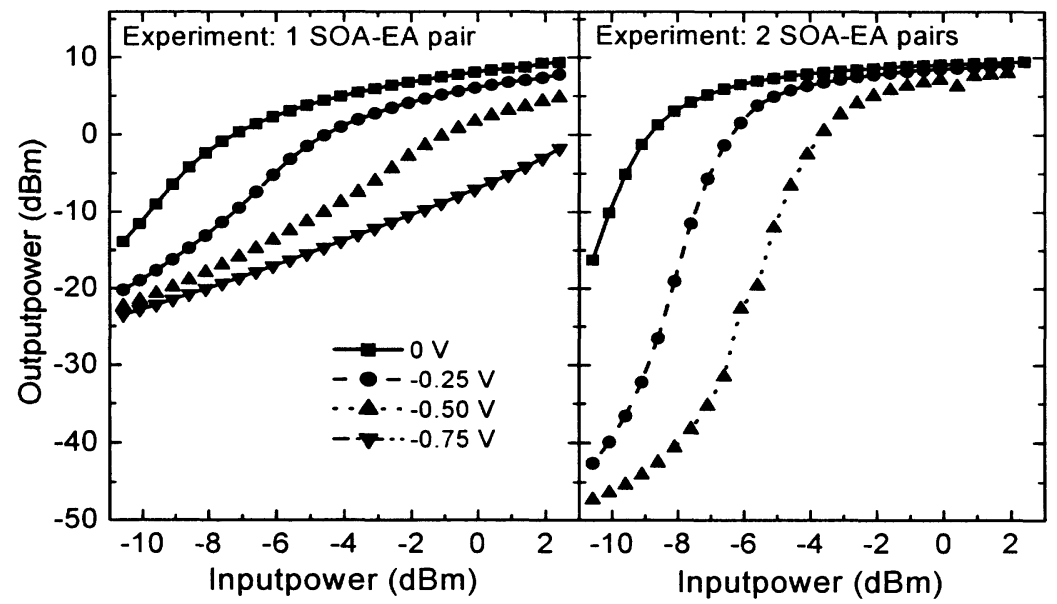

Fig. 3. Measured transfer functions for a single SOA-EA section (left) and two concatenated SOA-EA sections (right). The length of the SOAs is $545 \mu \mathrm{m}$ and the length of the EAs is $120 \mu \mathrm{m}$. The bias current to the SOA is $100 \mathrm{~mA}$ and the reverse bias of the first EA is varied, the second EA is unbiased.

Figure 4 shows modeling results corresponding to Fig. 3. We notice a good qualitative agreement in the behavior as a function of increased reverse bias. For low reverse bias there is almost no absorption in the EAs and the device acts as a saturable amplifier. For moderate fields the transfer functions become increasingly steeper until, at higher reverse bias, they again become more linear. We also see that the threshold increases with increased bias.

The lower nonlinearity of the calculated transfer functions as compared to the experimental results is attributed to our assumption of a constant (power independent) carrier lifetime (sweep-out time) and differential gain in the model, which also affects the saturation power since it is inversely proportional to these parameters. In the amplifier, the carrier lifetime and differential gain normally increases as the carrier density is reduced, i.e., as the input power is increased. In the absorber the sweep-out time increases with increasing carrier density, i.e. when the input power increases, due to carrier screening. These processes tend to decrease the saturation power of the devices as the input power increase and hence make the saturation characteristic sharper, an effect that is not included in the present model. The increase in steepness of the transfer functions as additional SOA-EA pairs are concatenated is seen in both the experimental and theoretical results. The main effect of concatenating several devices is an increase of the extinction ratio by increased suppression of the zero-level, whereas the one-level is almost unaffected. 


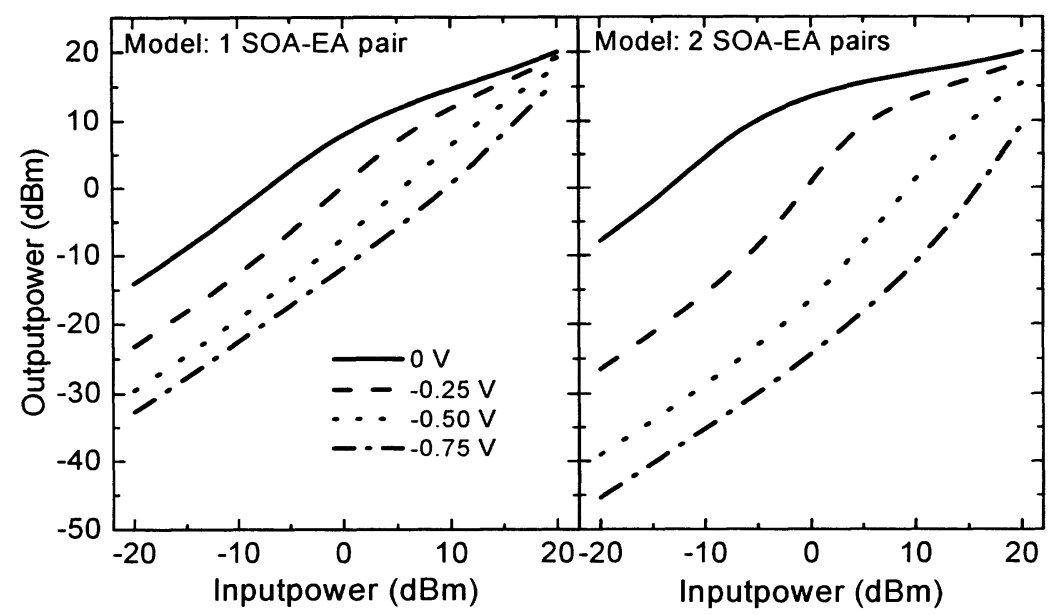

Fig. 4. Calculated transfer functions for a single SOA-EA section (left) and two concatenated SOA-EA sections (right). The gain, absorption and saturation powers used are extracted from measurements.

The influence of the reverse bias on the transfer functions is investigated in Fig. 5, where either the small signal absorption or the saturation power is changed while keeping the other parameter constant. The increase of the small signal absorption enables a larger degree of absorption bleaching and hence, a steeper nonlinearity. The increase of the EA input saturation power has the opposite effect, i.e. a large saturation power results in a nearly constant absorption without any bleaching and the nonlinearity remains small. Since the experimental results (not shown) show that for increasing reverse bias the EA input saturation power increases faster than the absorption, the flattening of the transfer function step as the reverse bias is increased can be explained.
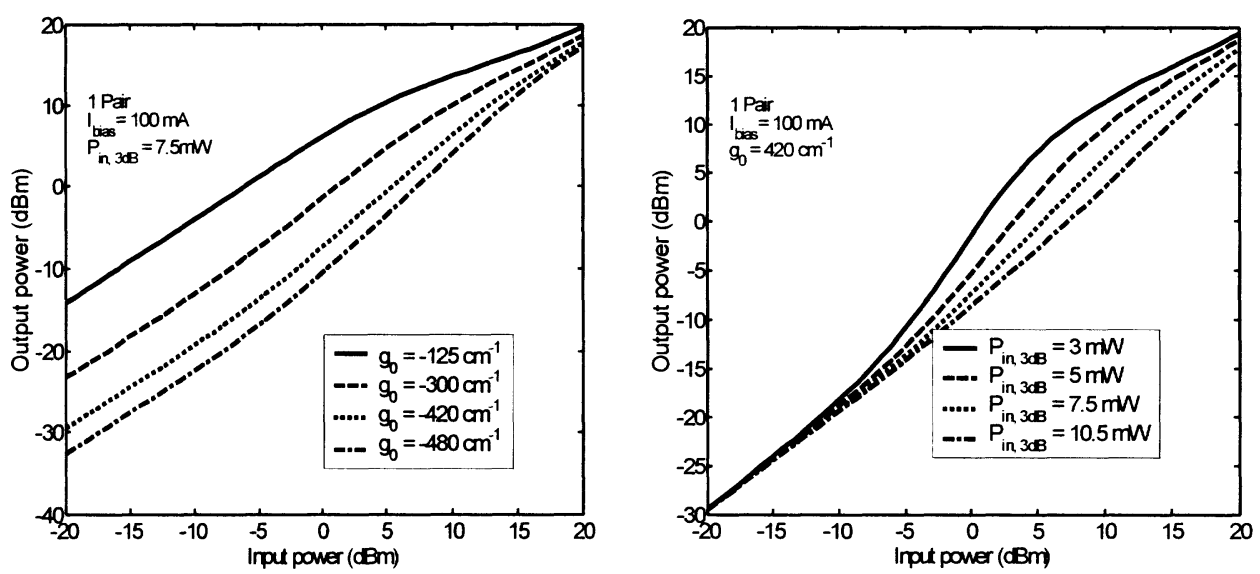

Fig 5. Calculated transfer functions when increasing the absorption (left) or the saturation input power (right) while keeping the other parameter constant.

\section{Conclusions}

We have experimentally and theoretically demonstrated steep and tunable power transfer characteristics of concatenated SOA-EA devices, which are well suited for 2R-regeneration. Theoretical modeling identified the origin of the nonlinearity as well as the bias dependence, forming the basis for further optimization.

\section{References}

[1] P. Öhlen and E. Berglind, "Noise accumulation and BER estimates in concatenated nonlinear optoelectronic repeaters", IEEE Photon. Technol. Lett. 9 1011-1013, (1997).

[2] L. J. Christiansen, L. Xu, K. Yvind, F. Öhman, L. Oxenløwe, and J. Mørk. "2R Regeneration in Concatenated Semiconductor Optical Amplifiers and Electroabsorbers". In Proceedings of ECOC 2004, 2004.

[3] F. Öhman et al., "Noise and regeneration in semiconductor waveguides with saturable gain and absorption”, IEEE J. Quantum Electron., 40, $245-255,(2004)$.

[4] C. Knöll et al. "Optimization of signal transmission by an in-line semiconductor optical amplifier-saturable absorber module", Opt. Commun., vol. $187(2001), 141-153$. 